\title{
Toward noninvasive assessment of flap viability with time-resolved diffuse optical tomography: a preclinical test on rats
}

Laura Di Sieno

Georges Bettega

Michel Berger

Cynthia Hamou

Marion Aribert

Alberto Dalla Mora

Agathe Puszka

Henri Grateau

Davide Contini

Lionel Hervé

Jean-Luc Coll

Jean-Marc Dinten

Antonio Pifferi

Anne Planat-Chrétien

\section{SPIE.}




\title{
Toward noninvasive assessment of flap viability with time-resolved diffuse optical tomography: a preclinical test on rats
}

\author{
Laura Di Sieno, ${ }^{a}$ Georges Bettega, ${ }^{b, c, d}$ Michel Berger, ${ }^{e}$ Cynthia Hamou,,${ }^{c, d, f}$ Marion Aribert, ${ }^{c, d}$ \\ Alberto Dalla Mora, ${ }^{a, *}$ Agathe Puszka, ${ }^{c, d, e}$ Henri Grateau, ${ }^{e}$ Davide Contini, ${ }^{a}$ Lionel Hervé, ${ }^{e}$ \\ Jean-Luc Coll,,${ }^{c, d}$ Jean-Marc Dinten, ${ }^{\mathrm{e}}$ Antonio Pifferi, ${ }^{\mathrm{a}, \mathrm{g}}$ and Anne Planat-Chrétien ${ }^{\mathrm{e}}$ \\ ${ }^{a}$ Politecnico di Milano, Dipartimento di Fisica, Piazza Leonardo da Vinci 32, 20133 Milano, Italy

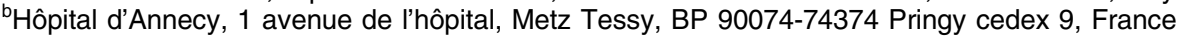 \\ 'University Grenoble Alpes-site santé, Institut Albert Bonniot, INSERM U1209, Domaine de la merci, 38000, La Tronche, France \\ ¿University Grenoble Alpes, BP53, 38041 Grenoble, France

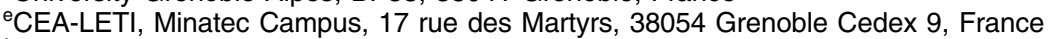 \\ ${ }^{f}$ Centre Hospitalier Universitaire-Grenoble, Boulevard de la Chantourne, 38700 La Tronche, France \\ IIstituto di Fotonica e Nanotecnologie, Consiglio Nazionale delle Ricerche, Piazza Leonardo da Vinci 32, 20133 Milano, Italy
}

\begin{abstract}
The noninvasive assessment of flap viability in autologous reconstruction surgery is still an unmet clinical need. To cope with this problem, we developed a proof-of-principle fully automatized setup for fast timegated diffuse optical tomography exploiting Mellin-Laplace transform to obtain three-dimensional tomographic reconstructions of oxy- and deoxy-hemoglobin concentrations. We applied this method to perform preclinical tests on rats inducing total venous occlusion in the cutaneous abdominal flaps. Notwithstanding the use of just four source-detector couples, we could detect a spatially localized increase of deoxyhemoglobin following the occlusion (up to $550 \mu \mathrm{M}$ in $54 \mathrm{~min}$ ). Such capability to image spatio-temporal evolution of blood perfusion is a key issue for the noninvasive monitoring of flap viability. @ The Authors. Published by SPIE under a Creative Commons Attribution 3.0 Unported License. Distribution or reproduction of this work in whole or in part requires full attribution of the original publication, including its DOI. [DOI: 10.1117/1.JBO.21.2.025004]
\end{abstract}

Keywords: image reconstruction techniques; light propagation in tissues; time-resolved imaging; tomography.

Paper 150595R received Sep. 3, 2015; accepted for publication Dec. 30, 2015; published online Feb. 2, 2016.

\section{Introduction}

Reconstructive surgery aims to restore anatomical defects with autologous tissues called "flaps." A flap includes the harvested tissues (skin, muscle, bone, etc., alone or combined) and their blood-supplying vessels (arteries and veins that constitute the pedicle) which are essential for the flap survival. In case of major reconstruction, the flap is completely separated from the donor site and the vessels are anastomosed (reconnected) to the vessels in the recipient site. In this case, surgeons execute the so-called "free-flap." Patency of the arterial and venous anastomosis is mandatory for free-flap survival. Human flap thickness is around 1.5 to $3 \mathrm{~cm}$ (in some cases, e.g., deep inferior epigastric flap, they can be thicker than $3 \mathrm{~cm}$ ) and vessels are buried deep below the skin, muscle, and fat layers.

The major complication of this type of surgery is thrombosis, ${ }^{1}$ i.e., vessel obstruction by a clot (usually a venous one) and it can lead to flap failure (necrosis). Even if this complication is not frequent (between 3\% to 7\% depending on the surgeon's experience and the type of flap ${ }^{2}$ ) the functional and esthetic consequences are dramatic if not treated rapidly. Salvage of the flap depends on the rapidity of restoration of the vessels permeability: $60 \%$ to $73 \%$ of complicated flaps could be salvaged if the thrombosis is treated before $6 \mathrm{~h}$ of evolution. ${ }^{3}$

*Address all correspondence to: Alberto Dalla Mora, E-mail: alberto. dallamora@polimi.it
Postoperative monitoring of the flap is commonly carried out by a clinical examination (inspection and palpation), a procedure that is highly operator-dependent. Moreover, this examination is even impossible when deep buried flaps are involved, e.g., in the case of mastectomy where an extra layer of biological tissue is used over the flap to reconstruct the volume of the breast or in the case of pharyngeal reconstruction. ${ }^{4,5}$ Importantly, changes in the appearance of the flap due to microvascular complications are delayed as compared to the instrumental detection and they will become visible only 1 or $2 \mathrm{~h}$ later.

Monitoring buried free flaps is difficult because the standard methods of clinical examination and surface Doppler monitoring are not possible. Buried free flaps, which represent less than $5 \%$ of all free flaps, are often monitored using an implantable 20-MHz ultrasonic Doppler probe. Implantable Doppler probe monitoring ${ }^{6}$ has a $100 \%$ sensitivity rate in detecting loss of flap perfusion, making it a good screening test for free-flap viability. However, it suffers from a high false-positive rate of $88 \%$, which results in a high proportion of subsequent negative surgical explorations. In addition, the use of implantable Doppler implies the need to perforate the flap which exposes it to the risk of infection and bleeding or vessel damage. Moreover, the quality and effectiveness of the monitoring depend on the stability of the probe position into the flap. Another solution consists of the externalization of a flap component, but it is often unaesthetic and it requires a supplementary surgery to remove the externalized part at the end of the monitoring period. 
Buried flaps could also be monitored by microdialysis, ${ }^{7}$ laser-Doppler flowmetry, ${ }^{8}$ or tissue oxygen monitoring. These methods are very sensitive but invasive. In this case also, there is a risk due to the need to implant a probe perforating the flap. In addition, similarly to implantable Doppler, the quality of the monitoring is very much dependent on the stability of the probe into the flap, which is sometimes difficult because of breath movements or in the neck because of deglutition. Moreover, most of these systems are expensive: in addition to the cost of the machine it is necessary to add the cost of the consumables that varies from 400 to $600 €$ per patient. Finally, monitoring is provided throughout the period during which the risk of vascular occlusion is the highest, i.e., four to six days following the operation, as frequently as possible-ideally continuously-but in practice every 4 to $6 \mathrm{~h}$. Since the chances of recovery (reperfusion of the flap) after the occlusion are directly proportional to the rapidity of diagnosis and subsequent surgical remedial treatment, an ideal continuous monitoring of flap viability is desired. In addition, it would be beneficial for the surgeon to have an idea of the position and the depth at which the occlusion is situated so as to better evaluate the more convenient and safer intervention to do.

In this context, "time-resolved near-infrared spectroscopy" appears to be an attractive technique to noninvasively assess tissues' oxygenation and deep perfusion thanks to its capability to encode depth in temporal delays of the time-resolved photon distributions. ${ }^{9}$ Indeed, photons that arrive later have traveled deeper, thus carrying information about oxygenation of deep layers. In recent years, it has been also demonstrated that the adoption of a small distance between the injection and collection fibers in reflectance geometry allows one to increase the spatial resolution and the overall number of detected photons without any loss in terms of depth sensitivity. ${ }^{10}$ Recently, we also showed that the use of a fast-gated single-photon avalanche diode (SPAD) coupled with a time-correlated single-photon counting (TCSPC) system permits to reach a dynamic range up to eight orders of magnitude ${ }^{11}$ (a limit set by the memory effect of silicon SPADs ${ }^{12}$ ), thus increasing retrieved information about deeper layers.

In a previous work, ${ }^{13}$ we presented a setup for diffuse optical tomography (DOT) based on fast-gated SPADs. High-dynamic range acquisitions were analyzed using the Mellin-Laplace transform, ${ }^{13}$ thus obtaining tomographic reconstructions of an absorbing inclusion within the turbid medium with better depth sensitivity with respect to classical free-running (i.e., "nongated") acquisitions. Also, we demonstrated that the adoption of short source-detector distances, combined with a fast-gated SPAD, can increase spatial resolution in DOT.

The goal of this work is to explore with a proof-of-principle test the suitability of an optical tomography system to assess flap viability. Indeed, DOT can provide a three-dimensional (3-D) reconstruction of oxy- and deoxygenated hemoglobin changes within the flap. This solution could allow surgeons to ideally continuously monitor the flap perfusion and, in case of complications, to plan the most suitable intervention.

To achieve this goal, we developed a more advanced setup for fast-gated tomography with respect to what was previously shown in Ref. 14. A fast wavelength multiplexing and the exploitation of the photon temporal distribution for a limited set of source-detector couples lead to an acquisition time for a full 3-D tomography of $3 \mathrm{~min}$, which is adequate for following the spatio-temporal evolution of oxy- and deoxy-hemoglobin.
As a first test, we used this setup to perform a preclinical study on abdominal fascio-cutaneous flaps in a rat undergoing a vein occlusion to simulate impairments in blood perfusion.

This paper is organized as follows: Sec. 2 deals with the experimental setup, measurement procedure, flap surgery, and data analysis; results are presented in Sec. 3, while in Sec. 4 we summarize the work and discuss the future perspectives.

\section{Material and methods}

\subsection{Experimental Setup}

A schematic of the setup is shown in Fig. 1. A supercontinuum fiber laser (Fianium LDT, London, UK) provided short light pulses at the repetition rate of $40 \mathrm{MHz}$. The illuminating source wavelength was selected by means of three interference filters (Coherent Inc.) centered at 750, 800, and $850 \mathrm{~nm}$ with a fullwidth at half maximum of $10 \mathrm{~nm}$. We selected those three wavelengths because of their particular position across the oxy- and deoxygenated hemoglobin spectrum (respectively, before, on, and after the isosbestic point). The filters were mounted on a motorized wheel which was controlled by a dedicated software to speed up the measurement. Light exiting from the interference filter was then attenuated by means of a motorized variable optical attenuator (VOA) and sent into an optical fiber switch (core size: $62.5 \mu \mathrm{m}$ ) so as to address six different output fibers. In the experiments presented in the following, in order to keep the measurement time to a minimum, only two output fibers were used to shine the sample into two different points of the flap [S1 and S2 in Figs. 1a and 2(a)].

Retro-diffused photons were then collected by means of two $400-\mu \mathrm{m}$ core optical fibers (one for each fast-gated SPAD module) at a source-detector distance of $10.6 \mathrm{~mm}$. Photons were then focused onto the SPAD (100- $\mu$ m diameter active area) of the fast-gated module, whose characteristics are described in Ref. 15.

The optical probe [see Fig. 1(b)] consisted of these optical fibers fixed in a $2.5-\mathrm{cm}$ diameter, $17.5-\mathrm{cm}$ long cylinder.

The synchronization signal provided by the laser was used to trigger the fast-gated SPAD modules - after a proper adjustable delay-and the two TCPSC boards (SPC 130, Becker\&Hickl $\mathrm{GmbH}$, Germany).

The fast-gated acquisitions were fully automatized by means of a dedicated software. For each wavelength, the delayer was set to three different values. This enabled to acquire the timeresolved curve in three different time regions and to optimize the count rate for each region in order to obtain a good signal-to-noise ratio, while keeping it within the single-photon counting statistics. ${ }^{11}$ For each delay, light was sequentially injected into two points of the probe using two different outputs of the switch. The attenuation introduced by the VOA was chosen in order to optimize the signal recorded from both detectors at the $10.6 \mathrm{~mm}$ interfiber distance.

\subsection{Measurement Procedures}

For each delay and injection point, retro-diffused photons were collected with both SPAD modules, with a measurement time of $5 \mathrm{~s}$. The total duration of a complete acquisition was about 3 min.

During the measurement, the probe was put on the flap [see Fig. 1(c)] and kept in place by a graduated mechanical support. We verified the repeatability of the probe placement by 

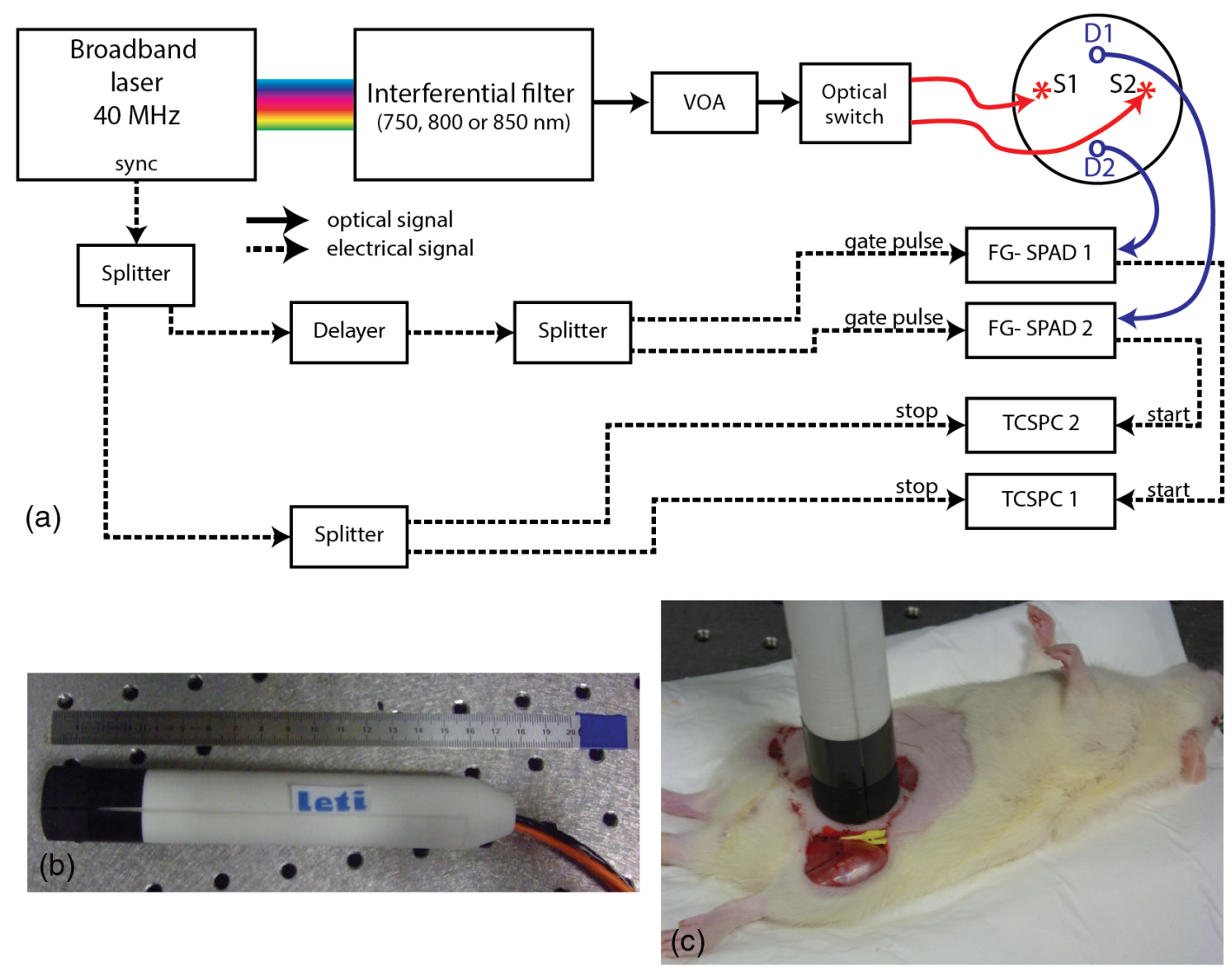

Fig. 1 (a) Schematics of experimental setup. VOA is variable optical attenuator; FG-SPAD is fast-gated SPAD module and in the probe the letters " $S$ " and " $D$ " indicate the source (injection) and detector (collection) fibers, respectively. (b) Picture of the probe. (c) Probe placed on the flap during the measurement.

removing and replacing it in the same position several times, obtaining a maximum variation of $5 \%$ among the different time-resolved acquisitions (data not shown). We also verified that small variations of the probe position (e.g., few millimeters) along the $z$-axis [see Fig. 2(a)] did not lead to significant changes in recorded time-resolved curves (data not shown), provided that there was no air gap between the probe and the flap. On the contrary, when the probe was moved down more than $4 \mathrm{~mm}$, we noticed a non-negligible variation in the time-resolved curve shape, possibly due to the local modification of both the blood concentration and tissues' structure. For this reason, we used the following procedure to place the probe. We first put the probe on the flap and visually checked the contact with the flap. Then we applied a slightly higher pressure (by moving the probe $2 \mathrm{~mm}$ down) in order to be sure that no air gap was present between the tips of the fibers and the flap. The pressure did not alter the blood circulation in the flap: proof of this is that no modification was measured on a control reference flap (without any clamp); moreover neither skin lesion nor arterial insufficiency was noted.

\subsection{Flap Surgery and Clinical Setup}

The rat abdominal fascio-cutaneous flap (constituted only by the skin and the underlying fascia with the nutrient vessels) was based on the superficial inferior epigastric bundle. The surgical technique applied in this paper is described in detail in Refs. 16 and 17. We choose this kind of flap since it is the most common animal model used for experimental flap monitoring.
Two adult female Wistar rats weighing from 250 to $350 \mathrm{~g}$ were operated on. They were in individual cages with free and unlimited access to both food and water. Fifteen days of acclimatizing in our institute's animal house were given to rats before they underwent surgery. On the first rat, two flaps were applied [see Fig. 2(a)], while on the second rat only one side Fig. 2(b) was operated on.

An inhalation of $4 \%$ isoflurane for $1 \mathrm{~min}$ was inducted, then the rats had a general anesthesia using $1.2 \mathrm{ml}$ intraperitoneal injection with a mixture of ketamine $(100 \mathrm{mg} / \mathrm{kg})$ and medetomidine $(100 \mu \mathrm{g} / \mathrm{kg})$. A $23 \mathrm{G}$ needle was entirely inserted with a 45 deg angle in the lower left or right quadrants of the animal's abdomen (on the opposite side to the flap), avoiding the upper quadrants and the median line at the base of the abdomen where the bladder is. Additional doses of anesthetic $(0.2$ to $0.3 \mathrm{ml})$ were administered according to the consciousness level of the animal during the surgical procedure. A test for response to pain was performed by heavy pressure on a leg every $30 \mathrm{~min}$.

After depilation of the abdomen and the thigh, the skin was prepared with an uncolored antiseptic solution. In order to prevent hypothermia, the rats were placed on their backs on a hot plate at $40 \mathrm{deg}$.

Each flap harvested half of the skin of the abdominal wall. It measured $\sim 35 \times 20 \mathrm{~mm}^{2}$ and was about 3- to 5-mm thick. The flap was elevated from the thorax to the thigh controlling the superficial epigastric pedicle on its deep side. With microsurgery tools and a $4 \times$ magnifier, the pedicle was then dissected and cleared and the vein was separated from the artery. The vein was surrounded by a nonobstructive thread loop made by 


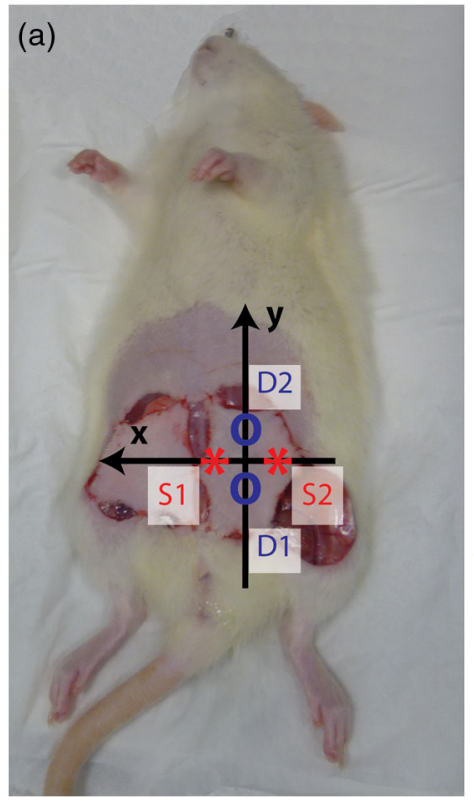

(b)

Fig. 2 (a) Rat after surgery: on the left flap are represented axes used for reconstruction maps presented in the followings and positions of injection ( $\mathrm{S} 1$ and S2) and collection fibers (D1 and D2). (b) Reconstructed volume with axes and representation of $x y$ and $y z$ planes.

a suture. The pedicle was clamped at the moment of the measures by the ligature of the suture. The skin flap was sutured (except in the groin crease) in its initial position [see Fig. 2(a)], leaving the pedicle accessible for later venous clamping.

The occlusion was complete as confirmed by the necrosis of the flap clinically evident at the end of the experiment as well as by the bluish discoloration of the flap. The arterial pressure was not monitored on these rats. Nevertheless, no modifications were observed on the flaps due to hemodynamic collapse (i.e., extremely low arterial pressure).

In the end, the rats were euthanized by an intracardiac injection of $150-\mu 1$ Dolethal.

This research was carried out in accordance with the "European convention on the protection of vertebrate animals used for experimental purposes or other scientific purposes" (Strasbourg, March 18th, 1986) and in agreement with the rules of the local ethical committee.

\subsection{Data Analysis}

At each measurement time, for each wavelength and each source-detector pair we used the fast-gated acquisition technique $^{18}$ in order to reconstruct a large dynamic range temporal point-spread function (TPSF). The reconstruction procedure is fully detailed in Ref. 13 .

The DOT image reconstruction method is described in Refs. 19 and 14. The direct problem was solved in 3-D using the finite volume method on a $4 \times 3 \times 3 \mathrm{~cm}^{3}$ mesh grid of regular steps of $0.1 \mathrm{~cm}$ in the $\mathrm{x}, \mathrm{y}$, and $\mathrm{z}$ directions [see Fig. 2(b)]. The extrapolated boundary conditions were implemented by enlarging the medium by $1 \mathrm{~mm}$ and imposing a null photon density at the extrapolated boundary. We adopted the Mellin-Laplace transform (MLT) approach, ${ }^{19}$ enabling time-windowing of the TPSF. Other time-windowing methods are proposed in the literature ${ }^{20,21}$ and discussed in Ref. 19.

We set the "precision" $(p)$ of the MLT equal to $3 \mathrm{~ns}^{-1}$, and $N=10$ as the number of MLT orders included in the reconstruction. Each wavelength $(\lambda)$ led to a 3-D absorption map $\mu_{a}(\lambda)$ that we used to compute the chromophore concentration maps. The decomposition in deoxy- and oxygenated hemoglobin (respectively, $\mathrm{HHb}$ and $\mathrm{HbO}_{2}$ ) was then retrieved for each pixel.

We choose the first measure after the occlusion as a reference in the reconstruction process, assuming for the model a homogeneous medium with $\mu_{a}=0.2 \mathrm{~cm}^{-1}$ and $\mu_{s}^{\prime}=10 \mathrm{~cm}^{-1}$, as derived by fitting the TPSF on a shaved rat with a homogeneous analytical solution of the transfer equation under the diffusion approximation ${ }^{22}$ with the extrapolated boundary condition. ${ }^{23} \mathrm{We}$ also used these values to initiate the 3-D absorption map at each wavelength. In this work, only absorption maps were reconstructed while the scattering coefficient was assumed constant for the whole measurement.

The solution was then approached by an iterative method, with the 3-D $\mu_{a}$ maps of the medium and the sensitivity matrix being updated at each step. We considered the stabilization of the $\mu_{a}$ values as convergence, which was reached in $<10$ iterations for all tested cases. The time needed to get the 3-D reconstruction on a standard $\mathrm{PC}$ is about $2 \mathrm{~min}$ without any particular optimization process.

\section{Results}

Figure 2 (a) depicts rat 1 after the flap surgery in both sides (left and right). On the left flap, the positions of injection and collection fibers are represented together with the orientation of $x$ - and $y$-axes used for the tomographic reconstruction. The $z$-axis refers to the depth, as can be seen in Fig. 2(b).

Figure 3 shows the reconstructed maps of variation in oxyand deoxygenated hemoglobin in the $y z$ and $x y$ planes, obtained for the left flap of rat 1 at different times from the reference measurement (start of the occlusion). The acquisitions are performed every $3 \mathrm{~min}$, starting immediately after the occlusion. The clamp on the vein was removed after 57 min (19 series of acquisitions). To reduce the number of panels, Fig. 3 shows 


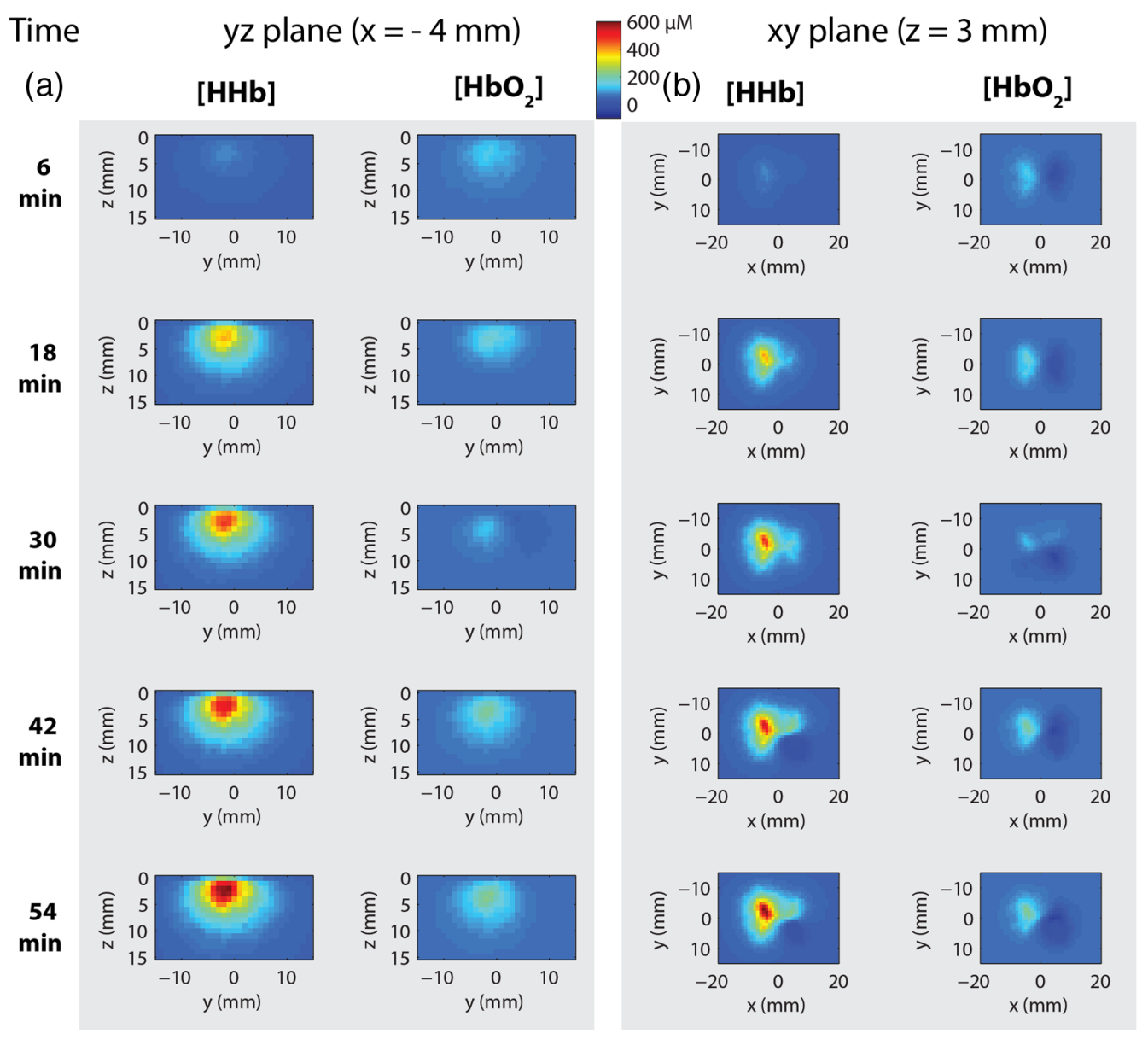

Fig. 3 Reconstructed maps of variation of oxy- and deoxygenated hemoglobin for the left flap of rat 1 at different times from the start of venous occlusion (see Video 1 for all times). (a) The left columns display the maps of $\mathrm{HHb}$ and $\mathrm{HbO}_{2}$ in the $y z$ plane (thus enlightening the depth distribution of chromophores) while (b) the right columns present the evolution of oxy- and deoxygenated hemoglobin in the $x y$ plane at a depth of $3 \mathrm{~mm}$. (Video 1, MPEG, $5.72 \mathrm{MB}$ ) [URL: http://dx.doi.org/10.1117/1.JBO.21.2.025004.1].

maps at steps of $12 \mathrm{~min}$. Maps for both planes at all times are presented in Video 1.

Figure 3(a) shows the evolution of $\mathrm{HHb}$ and $\mathrm{HbO}_{2}$ changes in the $y z$ plane [see Fig. 2(b)] for $x=-4 \mathrm{~mm}$. Looking at the maps, it is possible to notice that the $\mathrm{HHb}$ accumulation is mostly localized between the surface and a depth of $5 \mathrm{~mm}$, while the maximum increase of $\mathrm{HHb}$ is at $z=3 \mathrm{~mm}$. This value is reasonable, considering that the thickness of the flap is about 3 to $5 \mathrm{~mm}$. Small variations in concentration can be also noticed up to 10 to $15 \mathrm{~mm}$ in depth since, after the occlusion, the layer under the flap can also experience some modifications. In addition, the possible formation of a thrombus within the flap can increase its thickness, thus enlarging the volume where variations in oxygenation are detected. ${ }^{24}$

In reference to the time evolution, the accumulation of $\mathrm{HHb}$ starts 9 min after the clamping of the vein and increases with time. On the other hand, $\mathrm{HbO}_{2}$ concentration does not vary significantly with respect to $\mathrm{HHb}$ values. This is due to the fact that the arterial circulation was not massively altered by the specific vein occlusion. Even in the case of a total venous occlusion, part of the venous congestion can possibly be drained by the edge of the flap. The consequences on the arterial flow might be delayed causing the effect on $\mathrm{HbO}_{2}$ to appear later. It is also true that the small size of the flap (and consequently the small vascular flow) made it more difficult to identify flow alterations. However, this issue on flap dimensions is related to the use of rats, and will be overcome in the next studies with a model closer to humans.

In the two columns of Fig. 3(b), we show the reconstructed maps in the horizontal $x y$ plane at the depth $z=3 \mathrm{~mm}$, where the maximum $\mathrm{HHb}$ change was observed. After $6 \mathrm{~min}$ from the start of the occlusion, no significant accumulation of $\mathrm{HHb}$ is present, while after $18 \mathrm{~min}$, an increase of $\mathrm{HHb}$, can be clearly seen, which evolves in time. It is also possible to notice an increase of the area where $\mathrm{HHb}$ is concentrated. After $54 \mathrm{~min}$ the accumulation of $\mathrm{HHb}$ is higher and more spread out in the space as compared to the beginning. This phenomenon is most probably due to an accumulation of $\mathrm{HHb}$ that could not be washed out because of the occlusion. For that reason, the $\mathrm{HHb}$ concentration increased, leading to the formation of a thrombus. This hypothesis was verified after the euthanasia of the rat: the flap was unsewn, confirming the presence of a thrombus.

For $\mathrm{HbO}_{2}$, there is little accumulation in the same region where $\mathrm{HHb}$ concentration is increased, as already shown for the $y z$ plane.

In the $\mathrm{HHb}$ and $\mathrm{HbO}_{2}$ maps in the $x y$ plane, some artifacts may be observed: these are possibly due to both the geometry of the probe that was reduced in these experiments to four 
source-detector couples as well as to the assumption of a homogeneous medium as a reference state.

The temporal evolution of $\mathrm{HHb}$ and $\mathrm{HbO}_{2}$ up to 54 min after the vein occlusion is shown in Fig. 4 for three different pixels: one where the increase in $\mathrm{HHb}$ concentration is maximum (a), another where it is less pronounced (b), and the last where no major changes happened (c). The plots were extracted from the reconstructed 3-D maps at a depth of $z=3 \mathrm{~mm}$ and binning of adjacent pixels (bin factor: two).

Looking at the time evolution in Fig. 4, we can notice that for pixel a there is an increase in $\mathrm{HHb}$ which is steeper for the first $25 \mathrm{~min}$ while later it is much slower (this last part is probably due to the formation and then the slow increase in dimensions of the thrombus). On the other side, $\mathrm{HbO}_{2}$ does not vary significantly in its concentration.

In pixel $b$, the amount of $\mathrm{HHb}$ is quite stable throughout the whole experiment as well as the $\mathrm{HbO}_{2}$ content, which varies without following a particular trend.

In pixel c, no significant changes as compared to the background are present: fluctuations of $\mathrm{HHb}$ and $\mathrm{HbO}_{2}$ (see inset) are negligible with respect to the changes in $\mathrm{HHb}$ induced by the vein occlusion.

The experiment performed on the other flap (right) of the same rat displays a temporal and spatial trend similar to the one shown in Fig. 3 (see Video 1), just with a specular pattern, meaning that the maximum increase of $\mathrm{HHb}$ is observed on the right. This is consistent with the expected specular symmetry of blood perfusion in the left and right flaps.

The same overall behavior is observed in a second rat (see maps shown in Video 1), thus confirming the observed temporal and spatial evolution of concentrations of $\mathrm{HHb}$ and $\mathrm{HbO}_{2}$ after a venous occlusion.
The results obtained in all three flap experiments are consistent, thus allowing us to state that the proposed setup is able to properly detect the variation in perfusion after a venous occlusion, which mimics the possible complications arising after a flap surgery.

In these preclinical tests, we do not fully exploit all potentialities of the fast-gated high dynamic range acquisitions since the flap thickness on rats is pretty small ( 3 to $5 \mathrm{~mm}$ ) with respect to human flaps $(1.5$ to $3 \mathrm{~cm})$. However, the improvement in reconstruction given by the high dynamic range will be a crucial feature in future clinical tests on humans where blood vessels are deeper, thus requiring the better depth sensitivity given by fast time-gated technique ${ }^{10}$ to monitor the permeability of veins and arteries after flap surgery.

\section{Conclusions and Future Perspectives}

Monitoring flap viability is an unmet clinical need. Early diagnosis is needed in order to quickly act to save the flap by rapidly restoring the vessels' permeability. In this paper, we presented a new prototypal and fully automatized setup for fast time-gated optical tomography based on four source-detector couples. The aim of this work was to validate this setup for the noninvasive assessment of flap viability by means of preclinical in vivo measurements on rats. We demonstrated the possibility to get dynamic 3-D tomographic reconstruction of oxy- and deoxygenated hemoglobin thus detecting changes in concentrations caused by a venous occlusion. Indeed, a venous clamp is a relevant mode ${ }^{25}$ to simulate complications that can arise after flap surgery. In this case, since the vessels are not well reconnected, an increase in deoxygenated hemoglobin and the formation of a thrombus occurred.
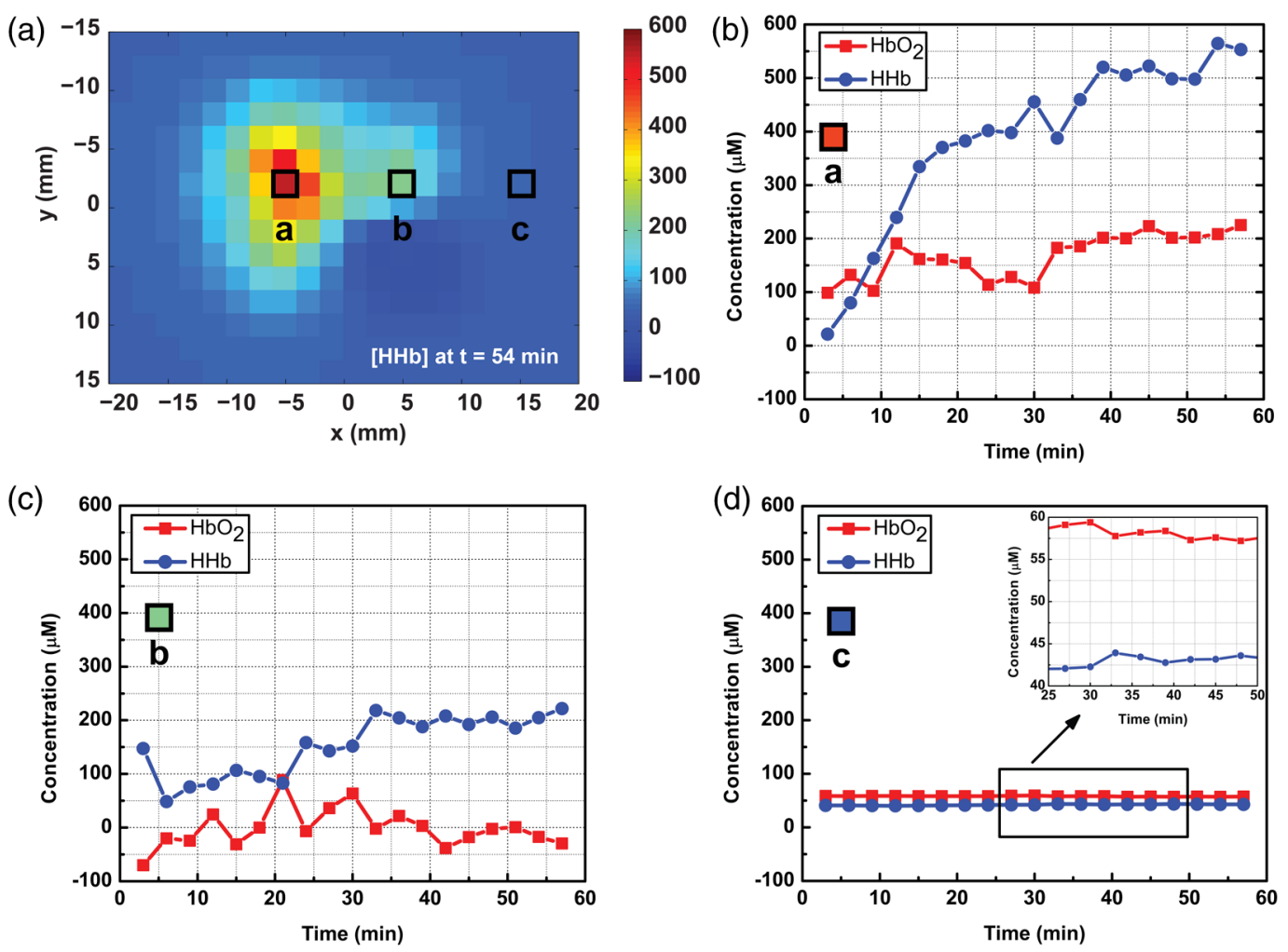

Fig. 4 Binned maps of $\mathrm{HHb}$ variation in concentration in the $x y$ plane (for $z=3 \mathrm{~mm}$ ) and time evolution of $\mathrm{HHb}$ and $\mathrm{HbO}_{2}$ concentrations in three different points (named $\mathrm{a}$, b, and c). 
At present, the acquisition time was about $3 \mathrm{~min}$ for a full 3-D image, which is already compatible with clinical needs (continuous check in the first $6 \mathrm{~h}$ and then measurements every $6 \mathrm{~h}$ for a few days). In the case of human flaps, the monitoring lasts for six days with control measurements every $6 \mathrm{~h}$ as routine. Considering the present clinical monitoring protocol, it is clear that our overall acquisition time is not a real concern. The total duration of a complete tomography is about $3 \mathrm{~min}$ and the time needed for the data analysis is about $2 \mathrm{~min}$ on a standard PC without any optimization process. Furthermore, in the future, sampling time can be reduced down to 1 min by reducing the dead time to a few seconds when changing the gate delay.

These positive results on the venous occlusion of superficial flaps in a rat encourage the further pursuit of this work. The next step is to tackle an animal model (e.g., pigs) closer to human flaps in terms of composition and thickness. Both venous and arterial occlusions should be investigated in this preclinical case before tackling clinical tests. In that situation, we will exploit at best the potentialities of the fast-gated SPAD since flaps will have thicknesses closer to the human case.

Future works will also consider the optimization of the probe and an increase in the number of source-detectors couples. This increase in measurement points will ameliorate 3-D reconstruction of oxy- and deoxygenated hemoglobin concentrations, thus possibly reducing artifacts in reconstructed maps and improving spatial resolution.

In addition, the development of low-cost and miniaturized detectors (e.g., silicon photomultipliers), ${ }^{26,27}$ compact light sources (e.g., vertical-cavity surface-emitting lasers), ${ }^{28}$ and improved electronics, replacing the expensive TCSPC instruments, ${ }^{29}$ will enable the realization of low-cost and high-performances diffuse optics devices opening the way to the clinical use of this technique.

\section{Acknowledgments}

Laura Di Sieno was supported by a scholarship of Région Rhône-Alpes (France). The research leading to these results has received funding from LASERLAB-EUROPE (Grant agreement No 284464, EC's Seventh Framework Programme). This work was partially supported by Cariplo Foundation under Grant No. 2013-0615. The authors acknowledge Julien Vollaire from INSERM U823 (Floralis, Institute Albert Bonniot, Grenoble, France) for having provided and prepared the rat experiments.

\section{References}

1. P. C. Neugan, "Monitoring techniques for the detection of flow failure in the postoperative period," Microsurgery 14, 162-164 (1993).

2. D. A. Kelly et al., "Impact of intraoperative vasopressor use in free tissue transfer for head, neck, and extremity reconstruction," Ann. Plast. Surg. 72, S135-S138 (2014).

3. Q. Yang et al., "The effect of early detection of anterolateral thigh free flap crisis on the salvage success rate, based on 10 years of experience and 1072 flaps," Int. J. Oral Maxillofacial Surg. 43, 1059-1063 (2014).

4. K. Yano et al., "Monitoring by means of color Doppler sonography after buried free DIEP flap transfer," Plast. Reconstr. Surg. 112, 1177-1178 (2003).

5. P. Yu et al., "Pharyngoesophageal reconstruction with the anterolateral thigh flap after total laryngopharyngectomy," Cancer 116, 1718-1724 (2010).

6. J. J. Rosenberg, B. D. Fornage, and P. M. Chevray, "Monitoring buried free flaps: limitations of the implantable Doppler and use of color duplex sonography as a confirmatory test," Plast. Reconstr. Surg. 118, 109-113 (2006).
7. J. Röjdmark, P. Hedén, and U. Ungerstedt, "Microdialysis-a new technique for free flap surveillance: methodological description," Eur. J. Plast. Surg. 21, 344-348 (1998).

8. J. C. Yuen and Z. Feng, "Monitoring free flaps using the laser Doppler flowmeter: five-year experience," Plast. Reconstr. Surg. 105, 55-61 (2000).

9. S. Del Bianco, F. Martelli, and G. Zaccanti, "Penetration depth of light re-emitted by a diffusive medium: theoretical and experimental investigation," Phys. Med. Biol. 47, 4131-4144 (2002).

10. A. Pifferi et al., "Time-resolved diffuse reflectance using small sourcedetector separation and fast single-photon gating," Phys. Rev. Lett. 100, 138101 (2008).

11. A. Dalla Mora et al., "Fast-gated single-photon avalanche diode for wide dynamic range near-infrared spectroscopy," IEEE J. Sel. Top. Quantum Electron. 16, 1023-1030 (2010).

12. A. Dalla Mora et al., "Memory effect in silicon time-gated single-photon avalanche diodes," J. Appl. Phys. 117, 114501 (2015).

13. A. Puszka et al., "Time-resolved diffuse optical tomography using fast-gated single-photon avalanche diodes," Biomed. Opt. Express 4, 1351-1365 (2013).

14. A. Puszka et al., "Spatial resolution in depth for time-resolved diffuse optical tomography using short source-detector separations," Biomed. Opt. Express 6, 1-10 (2015).

15. G. Boso et al., "Fast-gating of single-photon avalanche diodes with $200 \mathrm{ps}$ transitions and 30ps timing jitter," Sens. Actuators, A. 191, 61-67 (2013).

16. R. Gemperli and A. M. Munhoz, "The influence of type of vascular pedicle occlusion on the viability of skin island flaps: a postoperative quantitative assessment of flap survival in an experimental model in rats," Acta Cir. Bras. 28, 487-493 (2013).

17. J. Cubitt et al., "Reliable and reproducible murine models for commonly used abdominal plastic surgical flaps," J. Reconstr. Microsurg. 28, 161166 (2012).

18. A. Tosi et al., "Fast-gated single-photon counting technique widens dynamic range and speeds up acquisition time in time-resolved measurements," Opt. Express 19, 10735-10746 (2011).

19. L. Hervé et al., "Time-domain diffuse optical tomography processing by using the Mellin-Laplace transform," Appl. Opt. 51, 5978-5988 (2012).

20. M. Schweiger and S. R. Arridge, "Application of temporal filters to time-resolved data in optical tomography," Phys. Med. Biol. 44, 1699 (1999).

21. A. Liebert et al., "Time-resolved multidistance near-infrared spectroscopy of the adult head: intracerebral and extracerebral absorption changes from moments of distribution of times of flight of photons," Appl. Opt. 43, 3037-3047 (2004).

22. M. S. Patterson, B. Chance, and B. C. Wilson, "Time-resolved reflectance and transmittance for the non-invasive measurement of tissue optical properties," Appl. Opt. 28, 2331-2336 (1989).

23. R. C. Haskell et al., "Boundary conditions for the diffusion equation in radiative transfer," J. Opt. Soc. Am. A 11, 2727-2741 (1994).

24. S. Gioux et al., "First-in-human pilot study of a spatial frequency domain oxygenation imaging system," J. Biomed. Opt. 16, 086015 (2011).

25. A. Matsui et al., "Quantitative assessment of perfusion and vascular compromise in perforator flaps using a near-infrared fluorescence guided imaging system," Plast. Reconstr. Surg. 124, 451 (2009).

26. A. Dalla Mora et al., "Fast silicon photomultiplier improves signal harvesting and reduces complexity in time-domain diffuse optics," Opt. Express 23, 13937-13946 (2015).

27. E. Martinenghi et al., "Spectrally-resolved single-photon timing of silicon photomultipliers for time-domain diffuse optics," IEEE Photonics J. 7, 1 (2015).

28. A. Dalla Mora et al., "Towards next-generation time-domain diffuse optics for extreme depth penetration and sensitivity," Biomed. Opt. Express 6, 1749-1760 (2015).

29. L. Di Sieno et al., "Diffuse optics using a dual window fast-gated counter," Appl. Opt. 53, 7394-7401 (2014).

Laura Di Sieno received her master's degree in electronics engineering from the Politecnico di Milano, Italy, in 2011 and her PhD in physics from the same university in 2015. Since January 2015, she has 
been a postdoctoral fellow and her activity is mainly focused on the study and application of instrumentation for time-resolved optical spectroscopy of highly scattering media using single-photon avalanche diode enabled in fast-gated mode.

Georges Bettega is a professor of maxillofacial surgery, and he was the head of the Department of Plastic and Maxillofacial Surgery of Grenoble University Hospital. His works are focused on facial reconstructive surgery, bone regeneration, computer-assisted orthognathic surgery, and treatment of sleep apnea syndromes. He is working now in Annecy Genevois Hospital.

Michel Berger works as an engineer at CEA (Atomic Energy and Alternative Energies Commission, France) and has an expertise in design, characterization, and implementation of optical systems. For more than 15 years, he has worked on optical systems for clinical or preclinical applications such as fluorescence reflectance imaging, continuous wave diffuse optical tomography, time-resolved diffuse optical tomography, and spatial-resolved diffuse reflectance spectroscopy.

Cynthia Hamou is a plastic surgeon. She completed her surgical training in Paris, France. She also spent 2 years at Stanford University, where she did translational research focusing on developing genetically modified free flaps in a rat model.

Marion Aribert studied medicine at the Medical Faculty of Grenoble. After earning a Master 2 "Research and Cancer" (Lyon, 2015), she is currently in a third-year internship in reconstructive and cosmetic plastic surgery. She specializes in surgery of the hand and upper limb.

Alberto Dalla Mora graduated summa cum laude in electronics engineering from the Politecnico di Milano, Italy, in 2006. He received his $\mathrm{PhD}$ summa cum laude in information and communication technology from the same university in 2010 . Since 2011 , he has been an assistant professor at Dipartimento di Fisica of Politecnico di Milano. His research interests include time-resolved diffuse spectroscopy instrumentations and related applications.

Agathe Puszka obtained her Master of Science in physics from INSA Toulouse, France, in 2008. Since then she has been working in the field of biomedical optics, both in industry and in research. She obtained her PhD in physics from Université de Grenoble in 2013 thanks to her research on time-resolved diffuse optical tomography carried out at CEA, France.

Henri Grateau is a technician at CEA (Atomic Energy and Alternative Energies Commission, France). He conceives, designs, and produces equipment that will be used for the establishment and implementation of the measures studied devices.
Davide Contini received his master's degree in electronics engineering and his PhD in physics from the Politecnico di Milano, Milan, Italy, in 2003 and 2007, respectively. Since 2014, he has been an associate professor in physics at the Department of Physics, Politecnico di Milano. His research activity is mainly focused on the study of time-resolved spectroscopy of highly diffusive media for applications in biology and medicine.

Lionel Hervé is doing signal processing for medical imaging. He worked on x-ray absorptiometry by use of a spectrometric detector and on studies on the relationship between x-ray mammographic densities and cancer occurrence on large population trials at UCSF. He now works on the diffuse optical tomography (DOT) modality, in particular on time-resolved DOT, which consists in reconstructing the 3-D map of optical contrasts in highly diffuse media from optical measurements.

Jean-Luc Coll is director of research at INSERM and head of the Cancer Targets and Experimental Therapeutics research team of the INSERM Unit U823. His work is focused on the development of targeted molecules and optical-based instrumentation. His main interest is the introduction of optics for diagnostics and targeted treatment of cancer in clinical trials. In particular, his group is trying to develop systems for image-guided surgery.

Jean-Marc Dinten is a senior scientist at the Biology and Health Division in CEA-LETI. For more than 20 years, he has been developing medical image processing and reconstruction associated with the development of innovative x-rays and optical imaging and tomography systems. He now heads the Imaging Readout Systems Laboratory, which develops new optical imaging systems for health and biology applications.

Antonio Pifferi graduated in nuclear engineering in 1991 and received his $\mathrm{PhD}$ in physics in 1995 at the Politecnico di Milano. Since 2003, he has been an associate professor in the physics department of the Polytechnic of Milan. His research focuses on the development of new laser and instrumentation exploiting light propagation in highly scattering media with applications to optical characterization of biological tissues, and optical mammography.

Anne Planat-Chrétien has been project manager in the field of optical imaging applied to biology and health since 2000 at CEA-LETI, France. She is a PhD engineer in image processing-modeling, simulation, and algorithms. During the last decade, she has extended her expertise in system analysis, tests monitoring, and system characterization fields. Today she is leading the thematic on deep reconstruction of endogenous properties of biological tissues for both clinical and preclinical applications. 